\title{
On the Maximum Expected Electric Field in Electrically Small, Undermoded Enclosures
}

\author{
Paul Bremner \\ Robust Physics, Del Mar CA \\ Dawn Trout, Gabriel Vazquez Ramos, \\ NASA Kennedy Space Center, Cape Canaveral, FL
}


Common mode pick-up of conductors in a spacecraft fairing or avionics bay

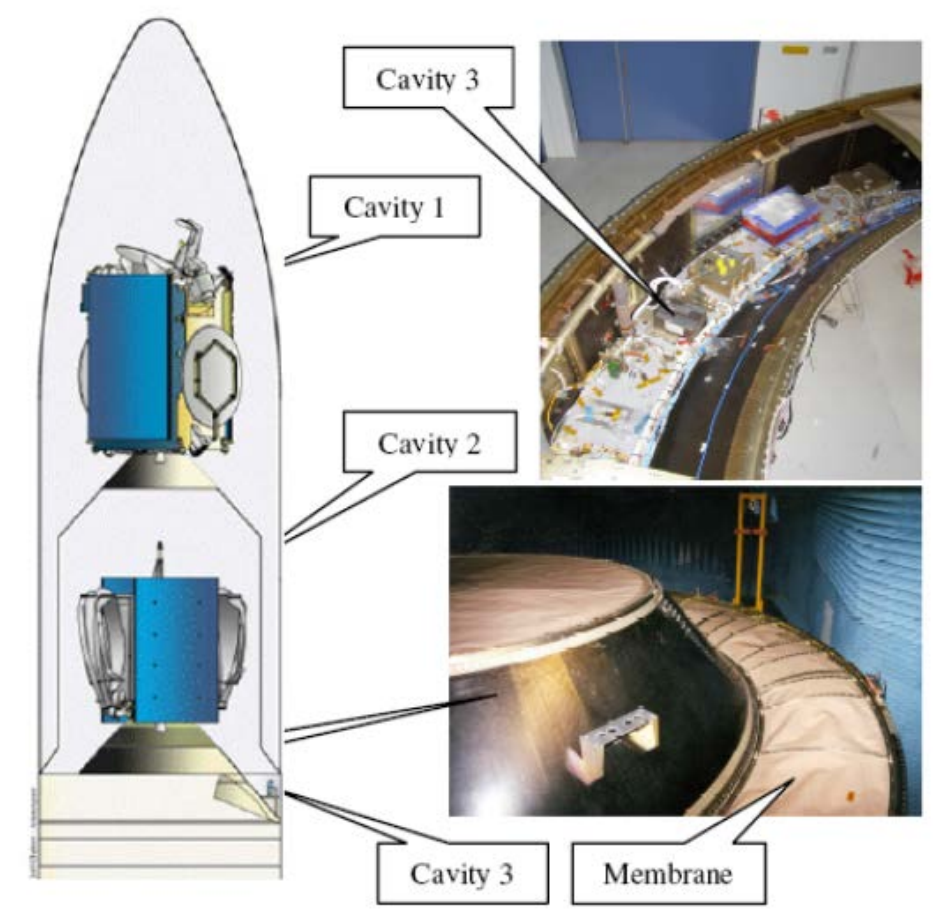

- Shaffar \& Gineste IEEE EMC 2011

\section{Surrogate system:}

Wire antenna in aperture box

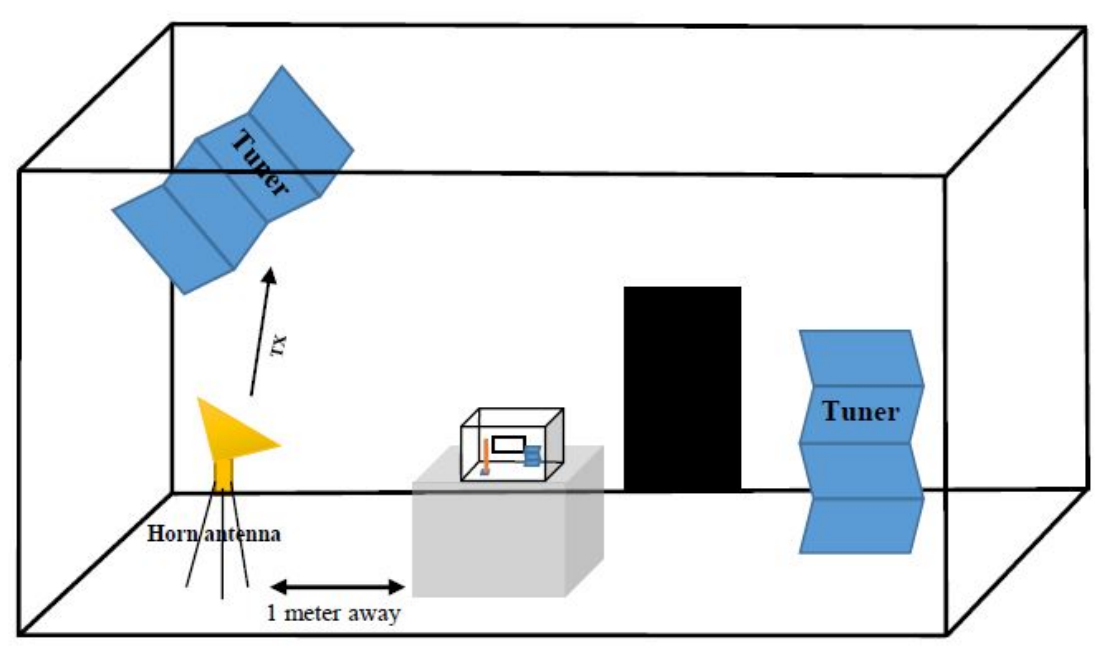

- Hill (NIST) 1994, 1996

- Holland \& St.John (AFRL) 1999, 2001

- Tait, Hager (NSWC Dahlgren) 2013

- Rajamani, West \& Bunting (OSU) 2014 


\section{Enclosure Test Configurations}

\section{3 different Apertures}

Wire Antenna in Aperture Box
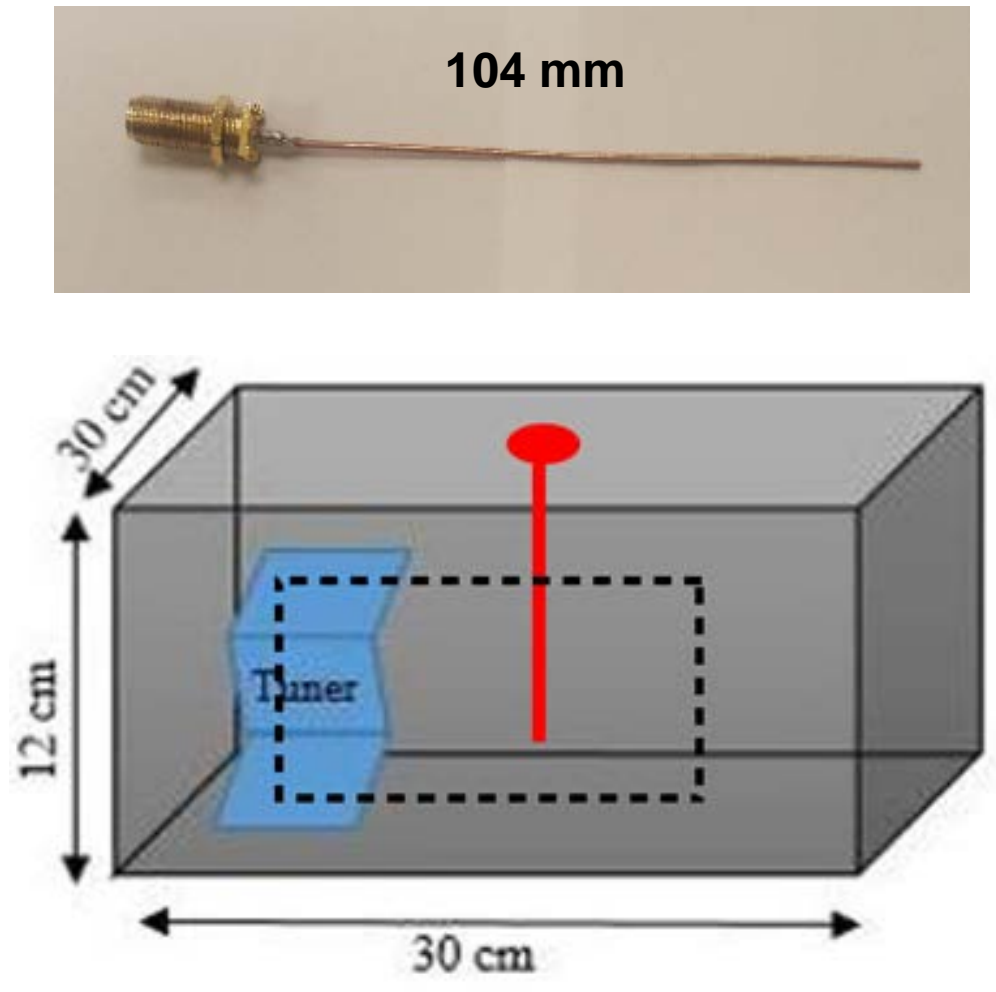

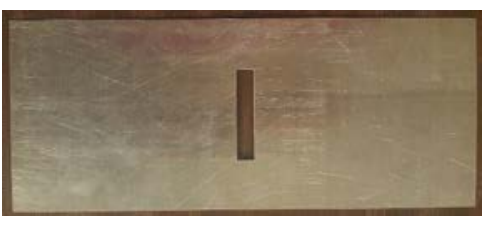

AP1 $1 \mathrm{~cm} \times 6 \mathrm{~cm}$

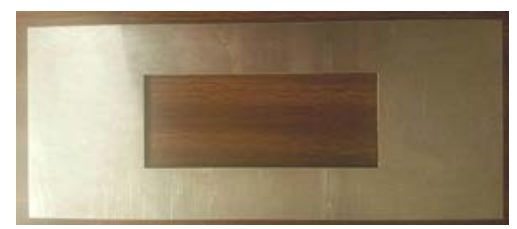

AP2 $60 \mathrm{~cm} \times 150 \mathrm{~cm}$

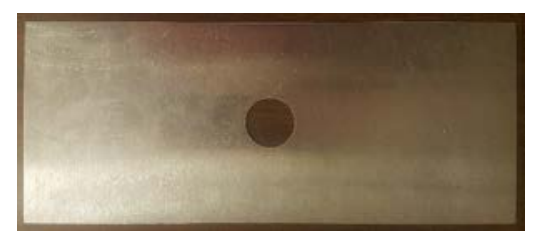

AP3 $30 \mathrm{~cm}$ dia 


\section{statistical mean of mode-strirred $E$ field}

Net Power input from Tx antenna

Power exchange through aperture

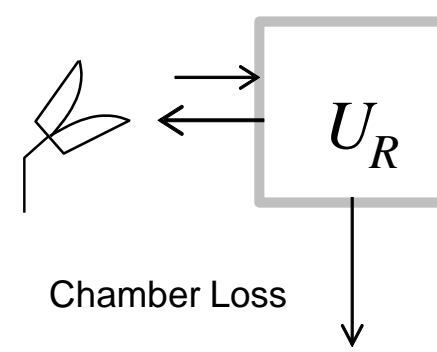

Net Power to Rx antenna
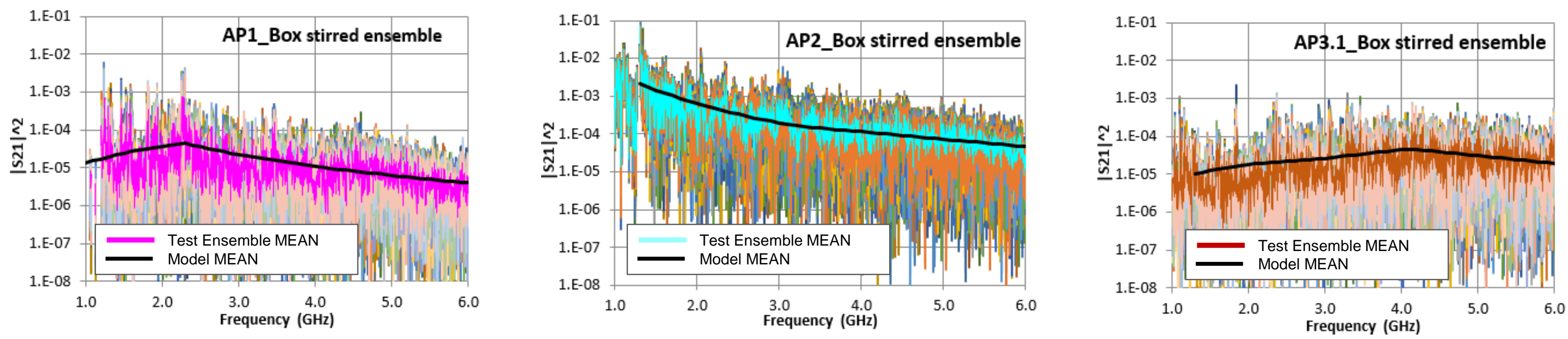

ESA Workshop on Aerospace EMC 20-22 May, 2019 Budapest Hungary

Robust Physics 


\section{Hill's Chi-sq. 2 dof Exponential distribution does not predict maximum $E$ field}

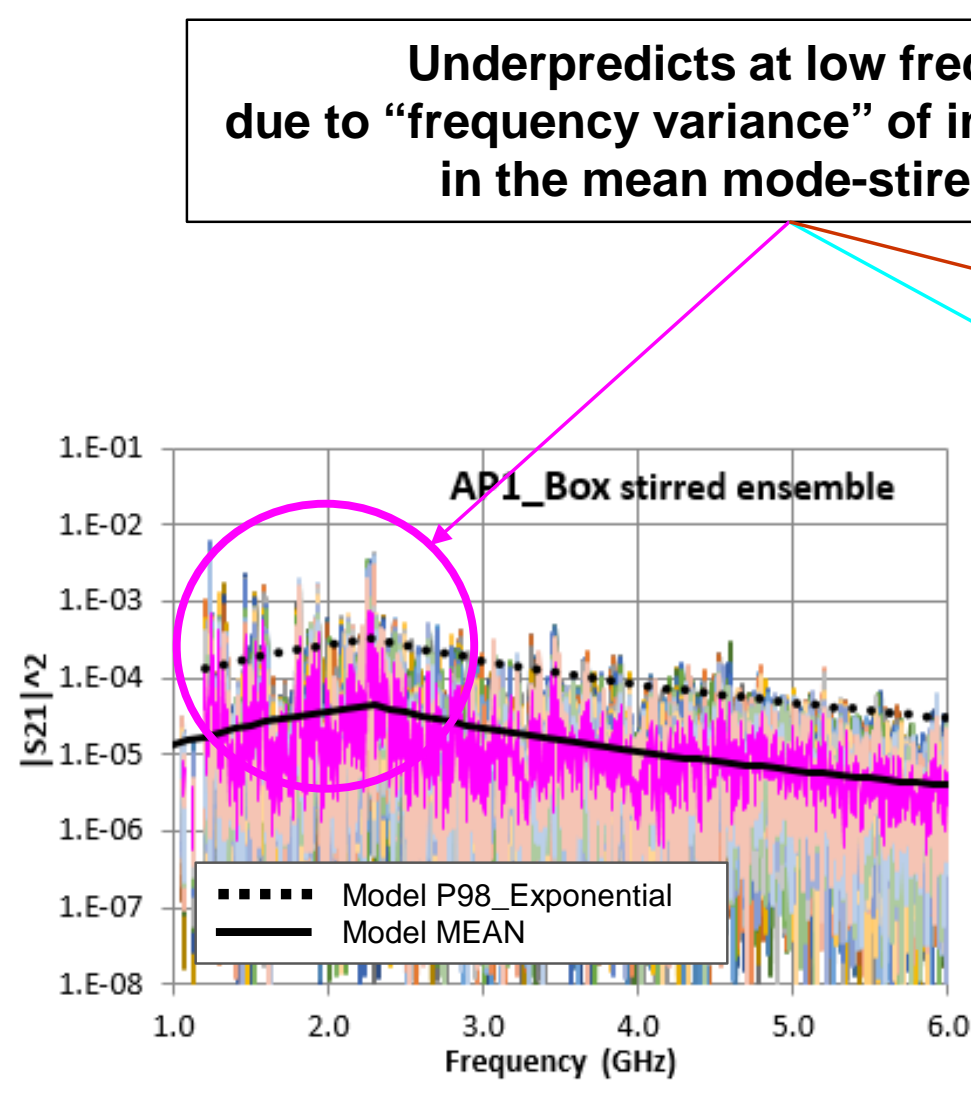

ESA Workshop on Aerospace EMC 20-22 May, 2019 Budapest Hungary
Max I Mean converges to Exponential at higher frequencies, lower frequency variance of individual modes

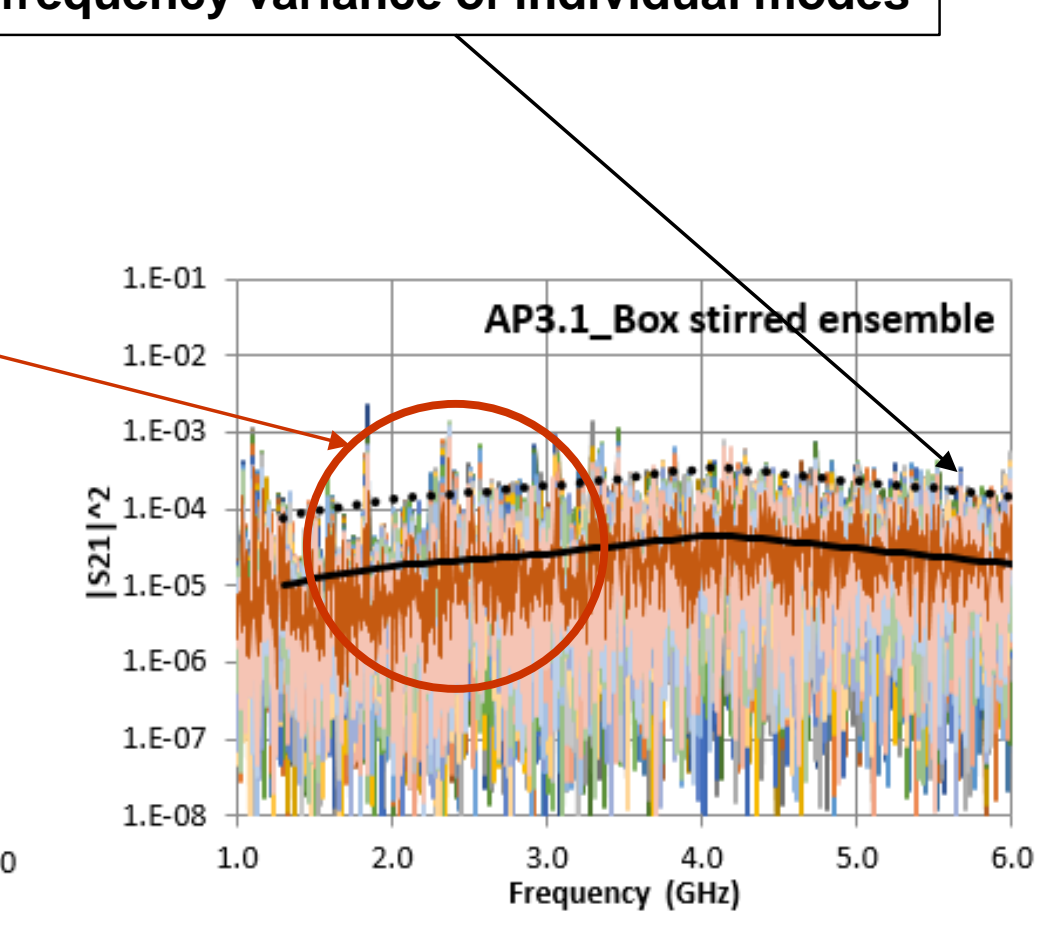


Numerical model of current received by wire in aperture box

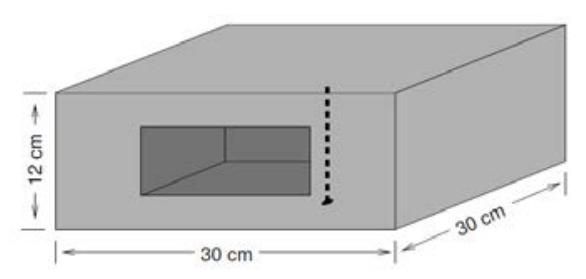

Resonant modes are widely spaced; response statistics need to be defined over frequency band

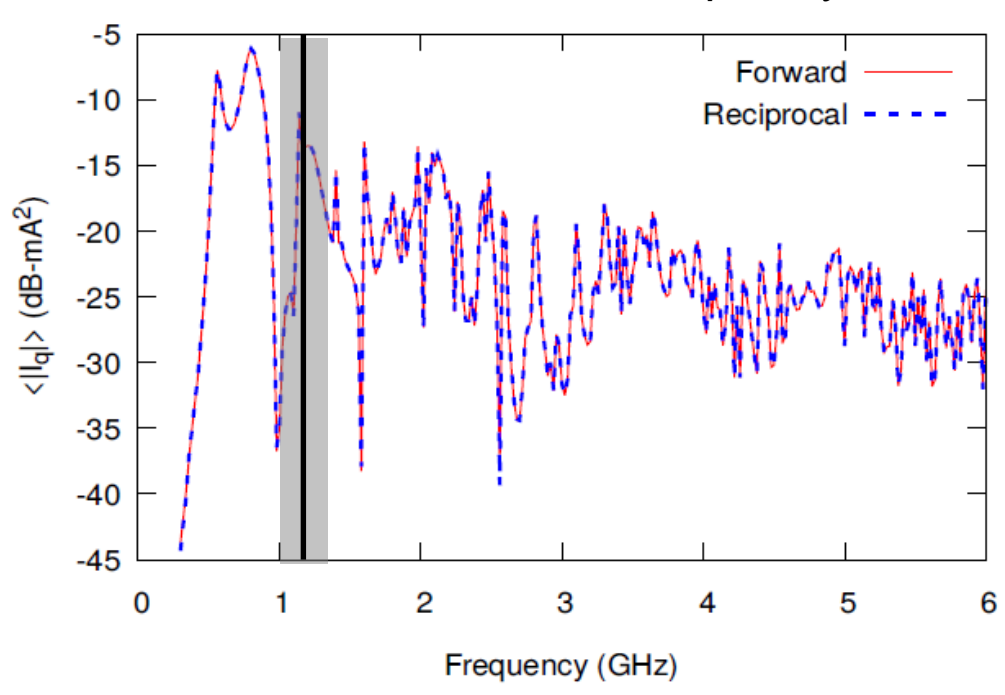

ESA Workshop on Aerospace EMC 20-22 May, 2019 Budapest Hungary

\section{Statistical distribution of wire current is} $\left|<S_{11}\left(f_{m}\right)>\right|^{2}$ weighted sum of Exponential PDFs

then [14, (4-74)]

The PDF of the frequency-stirred samples is

$$
\begin{aligned}
p_{S}(s)= & \frac{N_{1}}{N_{T}} p_{S \mid f}\left(s \mid f_{1}\right) \\
+ & \frac{N_{2}}{N_{T}} p_{S \mid f}\left(s \mid f_{2}\right) \\
& +\cdots+\frac{N_{M}}{N_{T}} p_{S \mid f}\left(s \mid f_{M}\right), \\
= & \sum_{m=1}^{M} \frac{N_{m}}{N_{T}} p_{S \mid f}\left(s \mid f_{m}\right),
\end{aligned}
$$

where $N_{T}=\sum_{m=1}^{M} N_{m}$ is the total number of samples.

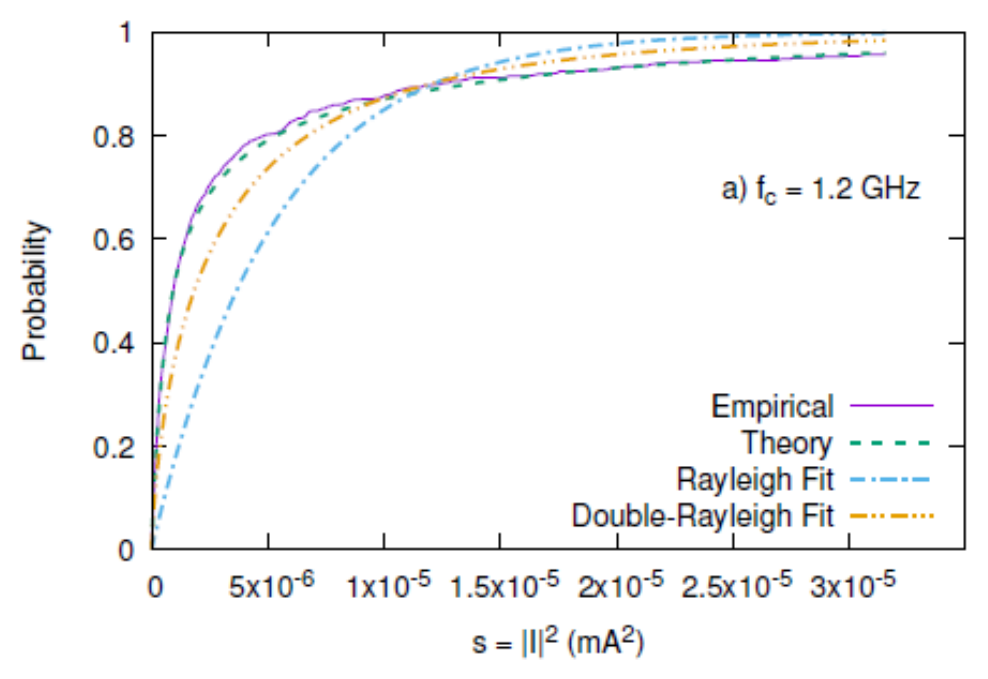


Ensemble of box-stirred modes (de-coupled from RC modes; no RC mode stirring)

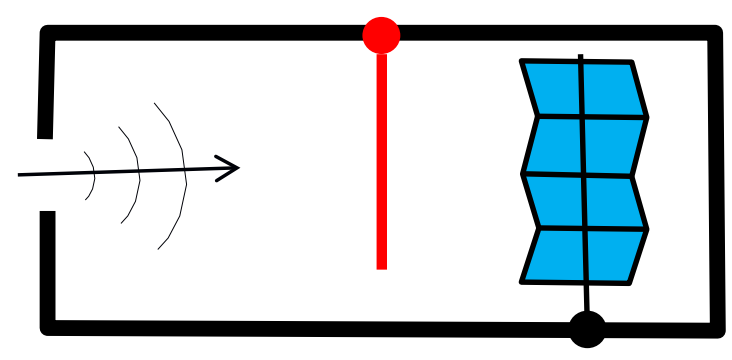

Power input deterministic for single box stirrer position:

- Effective source current at aperture (measured)

- Q of box modes (measured)

$$
\begin{aligned}
P_{i n}\left(\mathbf{x}_{i}, \omega\right) & =\frac{L_{d}^{2}}{\varepsilon V} \sum_{r} \frac{\omega \beta_{r} \omega_{r}^{2} \psi_{r}^{2}\left(\mathbf{x}_{i}, \xi\right) I_{\xi}^{2}\left(\mathbf{x}_{i}, \omega\right)}{\left[\left(\omega_{r}^{2}-\omega^{2}\right)^{2}+\beta_{r}^{2} \omega_{r}^{4}\right]} \\
& \equiv \sum_{r} a_{r}(\mathbf{x}) g_{r}(\omega)
\end{aligned}
$$

\section{Lyon variance model [JASA 1969]} based on statistics of mode spacing

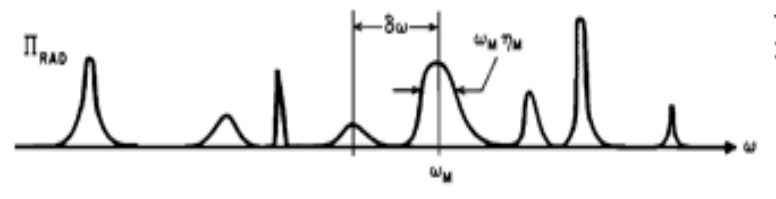

Figure 7. Graph of power received versus frequency $\omega$ for point excitation of a multi-modal system

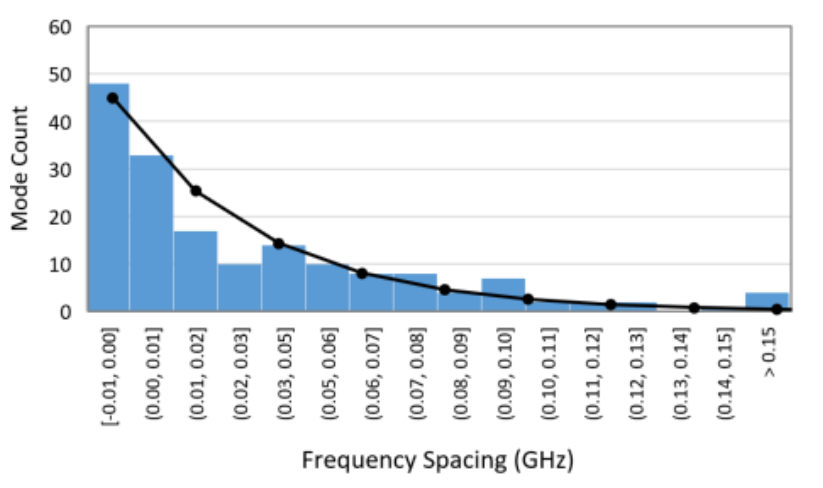

Relative variance of Power input

$$
r^{2}\left(P_{\text {in }}\right)=\frac{\sigma_{P}^{2}}{\left\langle P_{\text {in }}\right\rangle}=\frac{1}{2 m} \frac{\left\langle\psi_{r}^{4}\right\rangle}{\left\langle\psi_{r}^{2}\right\rangle^{2}}
$$

Relative variance on mean-squared Electric field

$$
r^{2}\left(|E(\mathbf{x}, \omega)|^{2}\right)=1+\frac{1}{2 m} \frac{\left\langle\psi_{r}^{4}\right\rangle^{2}}{\left\langle\psi_{r}^{2}\right\rangle^{4}}=1+\frac{5.7}{m}
$$

where $m=$ Modal Overlap 


\section{Modal Overlap}

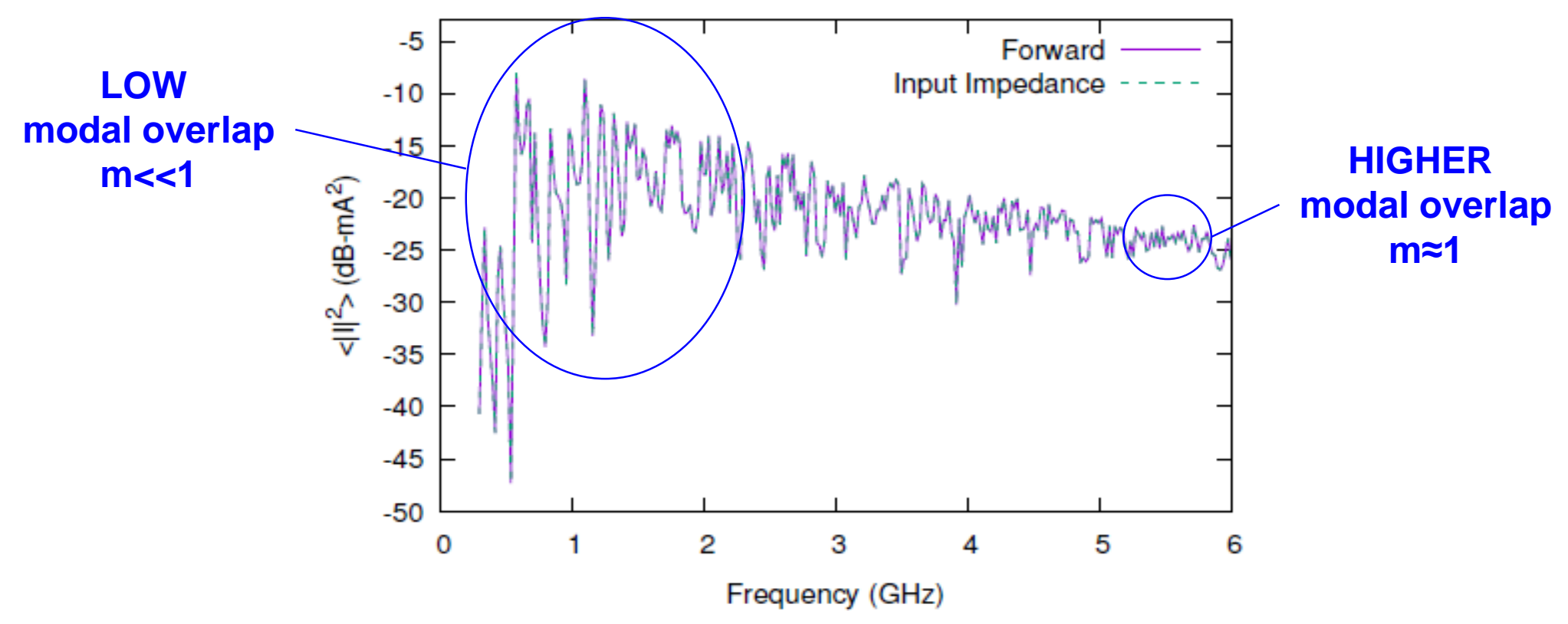

Modal Ovelap $=$ Ratio of Modal $\mathrm{Q}$ Bandwidth to Average Frequency spacing of Resonant Modes

$$
m(\omega)=\pi \omega n / 2 Q
$$

where $n(\omega)=\omega^{2} V / \pi^{2} c^{3}$ is modal density and frequency spacing $d f=1 / 2 \pi n(\omega)$ 


\section{Statistical model for Relative Variance of EM field Energy level}

Lyon model for Poisson mode spacing statistics and infinite ensemble

$$
r^{2}\left(P_{\text {in }}\right)=\frac{\sigma_{P}^{2}}{\left\langle P_{\text {in }}\right\rangle}=\frac{1}{2 m} \frac{\left\langle\psi_{r}^{4}\right\rangle}{\left\langle\psi_{r}^{2}\right\rangle^{2}}
$$

Weaver enhancement for Gaussian Orthogonal Ensemble (GOE) statistics and finite sample ensemble

$$
r^{2}\left[\left\langle|E|^{2}\right\rangle\right]=\frac{1}{L N}+\frac{1}{2 m}\left\{\left(\frac{K}{N}+1-\frac{1}{N}\right)\left(\frac{K}{L}+1-\frac{1}{L}\right)-\frac{2}{L N}-1\right\}
$$

where $L$ and $N$ are respectively the number of receiver and source positions used to calculate cavity energy

\section{NIST measurement of LaRC Reverb chamber} Bremner IEEE EMC 2015

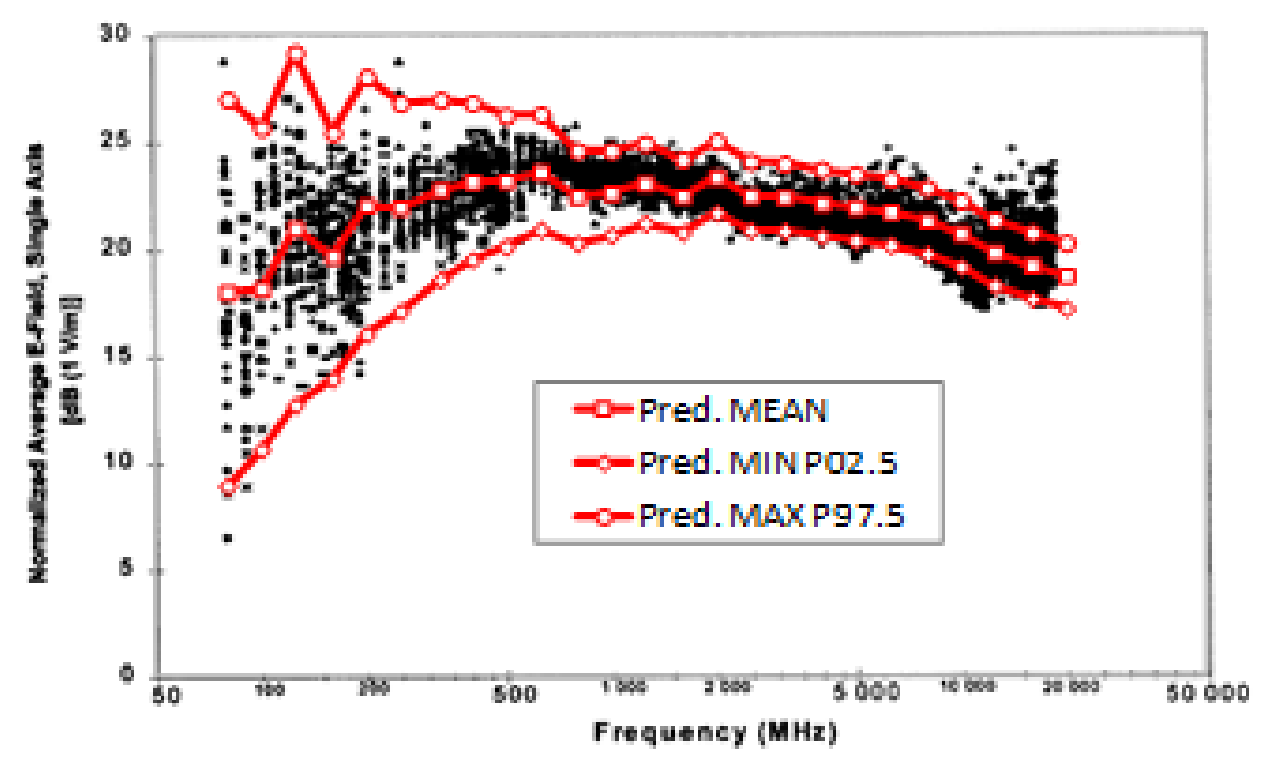

Figure 9. Maximum [P99.5] and minimum [P2.5] of Avg[Er] predicted by (14), compared with measurements in LaRC chamber $B$ (reproduced from [12], [3]) 


$$
r^{2}\left(|E(\mathbf{x}, \omega)|^{2}\right)=1+\frac{1}{2 m} \frac{\left\langle\psi_{r}^{4}\right\rangle^{2}}{\left\langle\psi_{r}^{2}\right\rangle^{4}}=1+\frac{5.7}{m}
$$

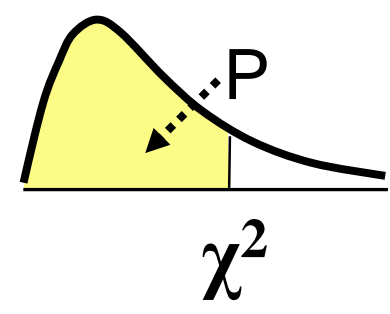

Mode shape PDF Rayleigh (Exponential)

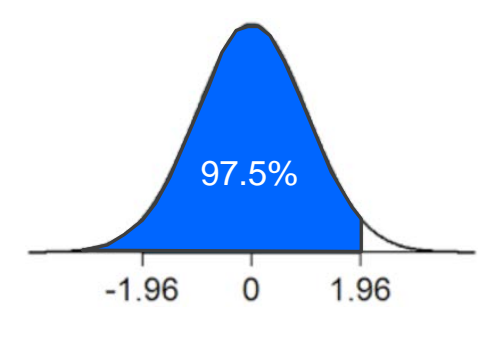

Modal Frequency statistics Log Normal PDF

$$
\operatorname{Max}\left[\left|S_{21}\right|^{2}\right]=\operatorname{Max}_{\text {Freq }}\left[\left|S_{21}\right|^{2}\right]+P_{\text {Rayleigh }}\left[\left|S_{21}\right|^{2}\right]
$$

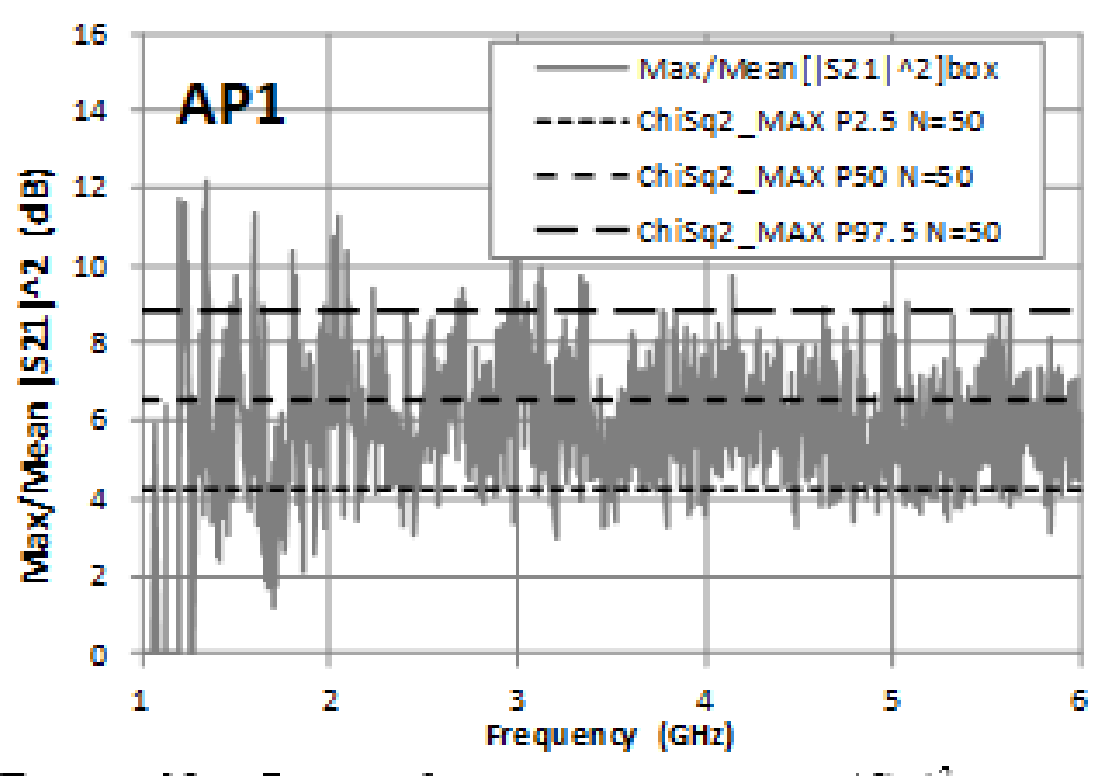

Figure 12. Ratio of maximum to mean $\left|S_{21}\right|^{2}$ at each frequency, band average and expected ratio of exponential distribution with ensemble $N=50$ 


$$
\begin{gathered}
r^{2}\left(|E(\mathbf{x}, \omega)|^{2}\right)=1+\frac{1}{2 m} \frac{\left\langle\psi_{r}^{4}\right\rangle^{2}}{\left\langle\psi_{r}^{2}\right\rangle^{4}}=1+\frac{5.7}{m} \\
\operatorname{Max}\left[\left|S_{21}\right|^{2}\right]=\operatorname{Max}_{\text {Freq }}\left[\left|S_{21}\right|^{2}\right]+P 98_{\text {Rayleigh }}\left[\left|S_{21}\right|^{2}\right]
\end{gathered}
$$
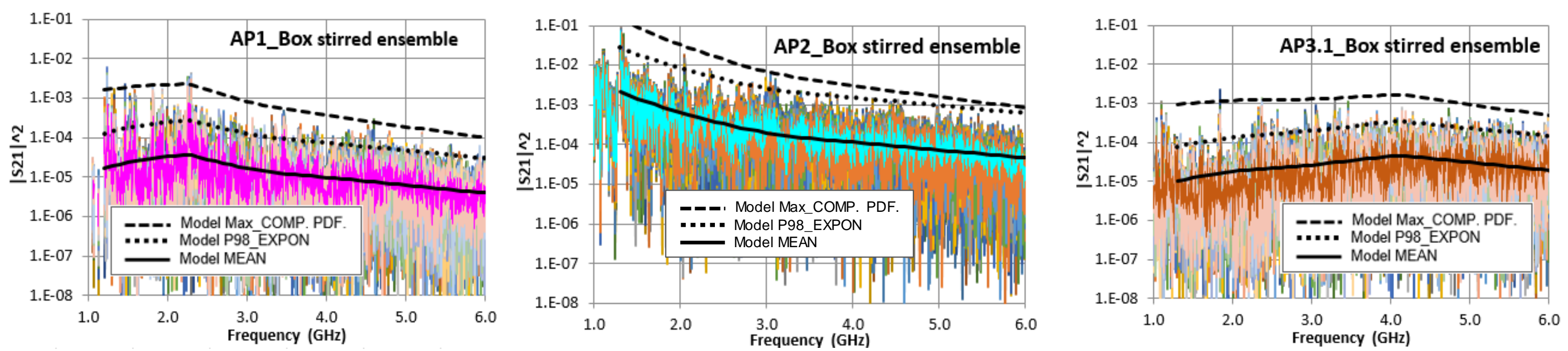

ESA Workshop on Aerospace EMC 20-22 May, 2019 Budapest Hungary 


\section{Conclusions}

- Hill power balance model is robust for frequency- \& space-averaged E field prediction

- Both undermoded and overmoded enclosures

- Variance and Max Expected E field in undermoded enclosure not predicted by Rayleigh statistics

- Additional "frequency variance" due to widely spaced modal responses

- West [IEEE EMC 2018] provides deterministic method to calculate Variance, PDF \& Max Expected

- Lyon [JASA 1969] provides an alternative "frequency spacing statistics" model for Variance

- Max Expected E field response of undermoded enclosures

- Frequency variance at low frequencies; Rayleigh statistics at high frequency

- Authors postulate a PDF that transitions from LogNormal to Exponential

- Trends match measured data 


\section{Back-up material}




\section{Modal Q estimation Sensitivity to $S_{11}$ measurement location}

Position $4 \quad$ Position 3
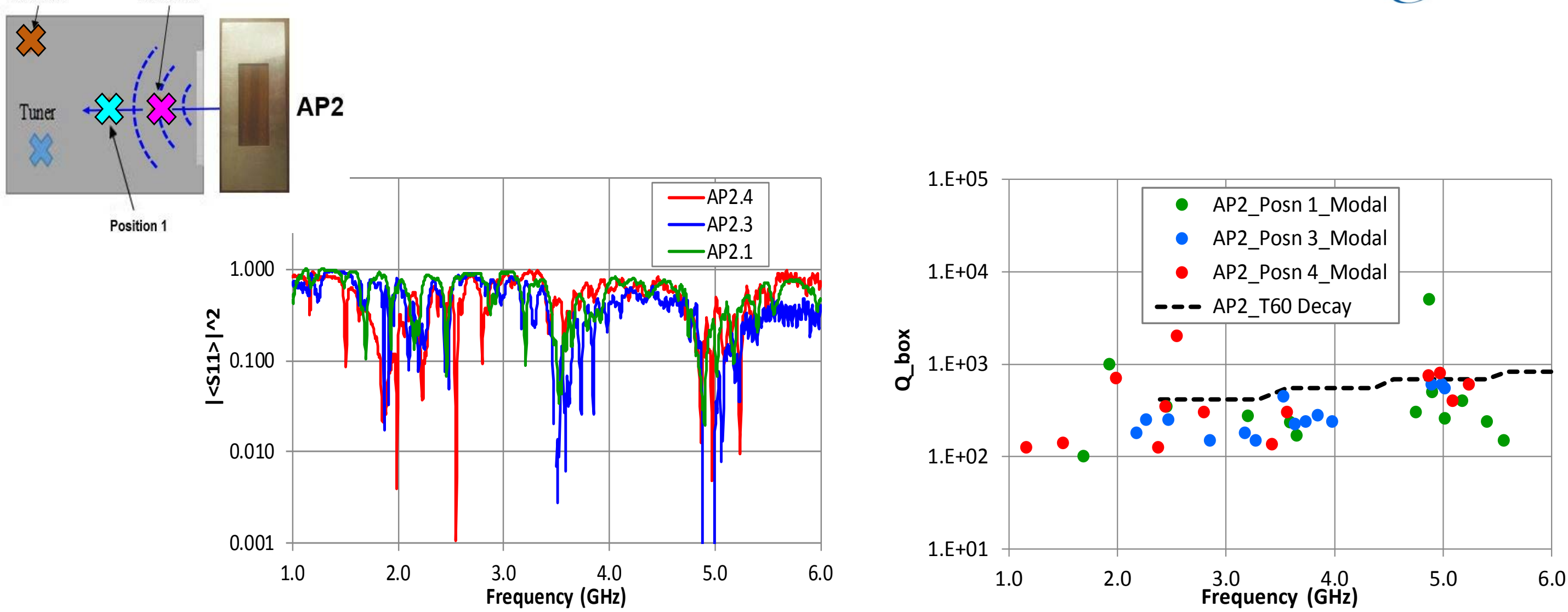


\section{Ideal Antenna}

$$
Q_{a n t}=\frac{16 \pi^{2} V_{c}}{\lambda^{3}}
$$

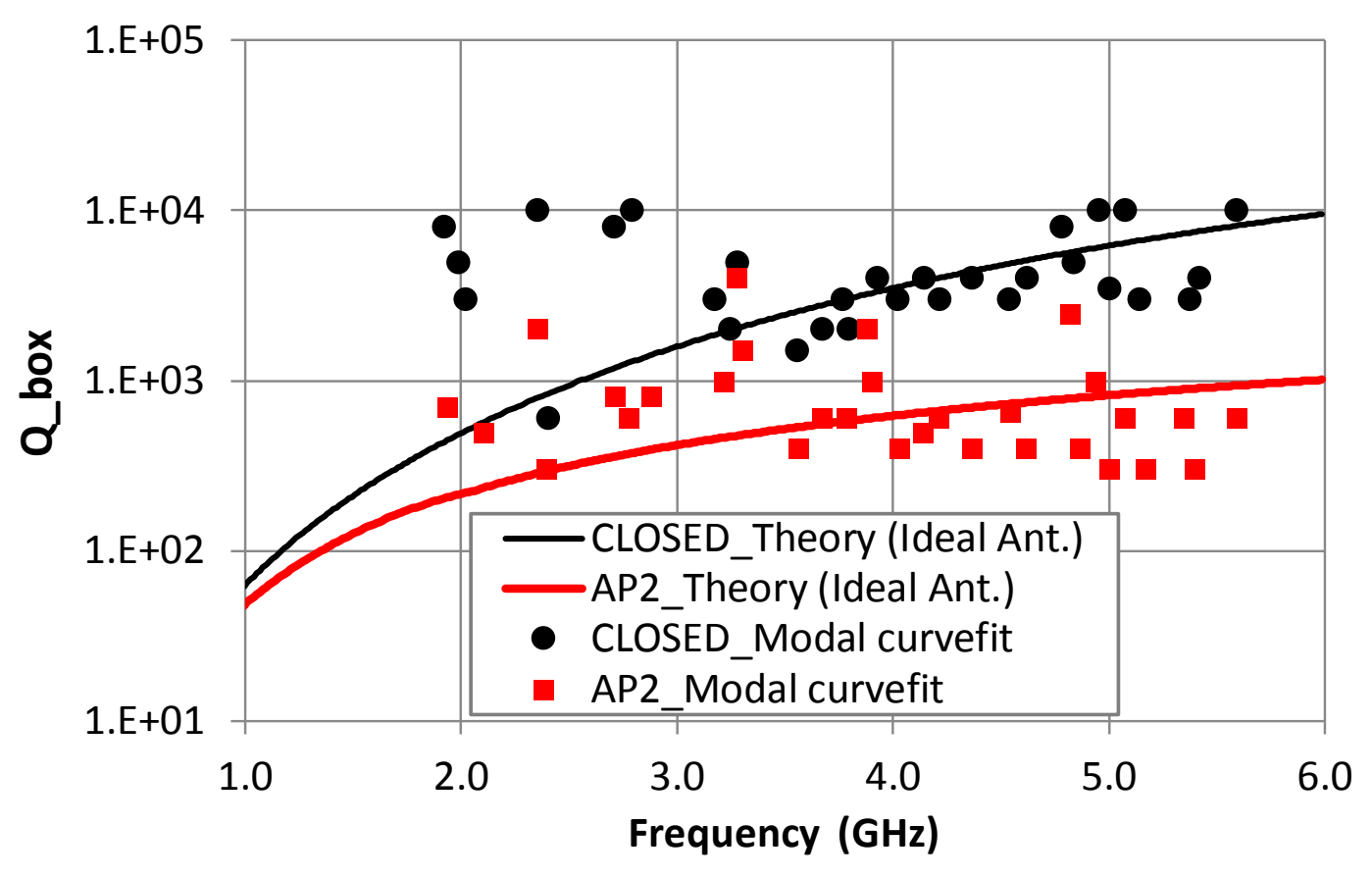

\section{Wire Antenna}

$$
Q_{a n t}=\frac{16 \pi^{2} V_{c}}{\left(1-\left|\left\langle S_{11}\right\rangle\right|^{2}\right) \lambda^{3}}
$$

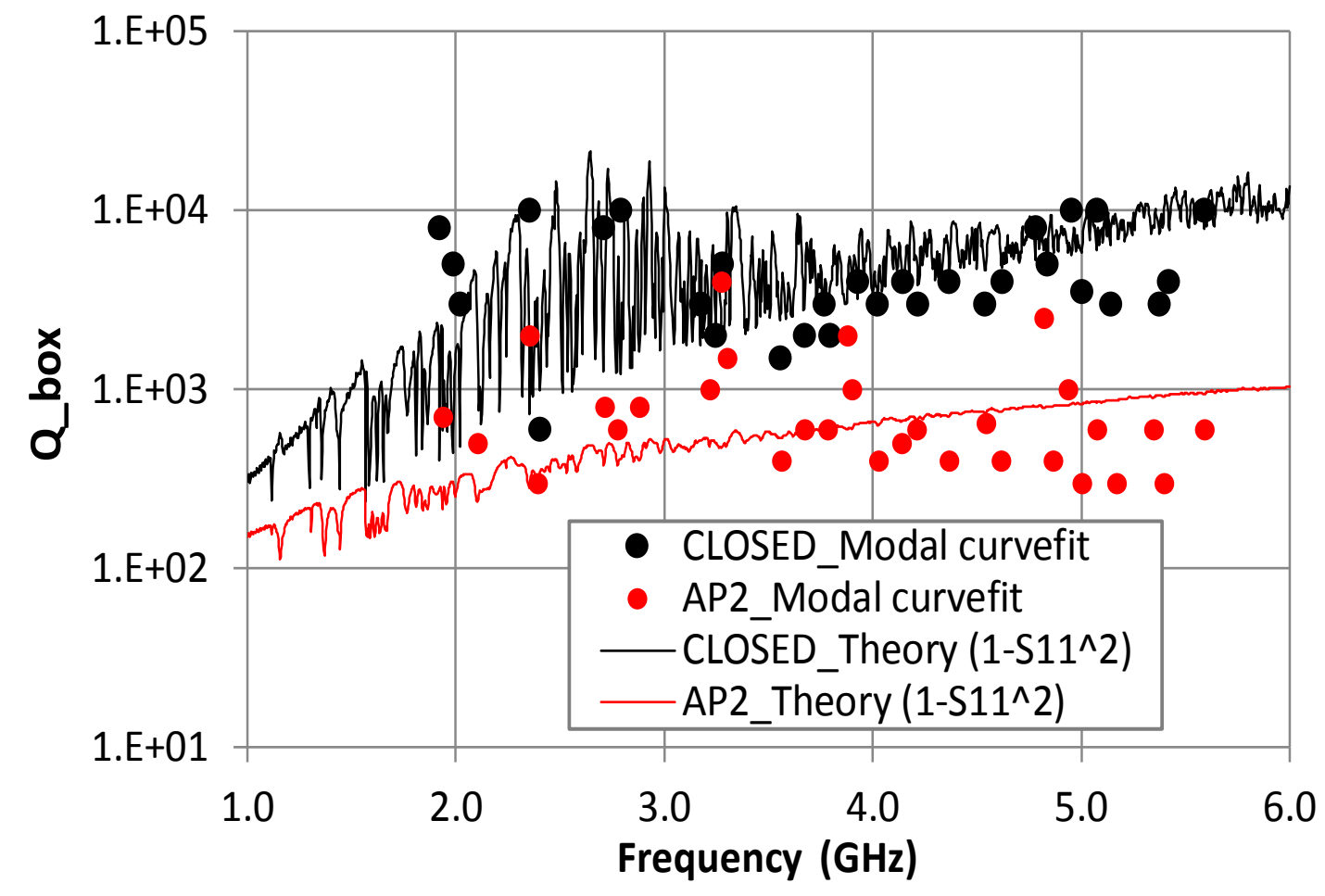

